\title{
Awareness of Antimicrobial Prophylaxis for Infective Endocarditis Among Dental Students and Interns at a Teaching Hospital in Jeddah, Saudi Arabia
}

\author{
Maha A. Bahammam ${ }^{1, *}$ and Noha M. Abdelaziz ${ }^{2}$ \\ ${ }^{I}$ Department of Periodontology, Faculty of Dentistry, King Abdulaziz University, Jeddah, Saudi Arabia; ${ }^{2}$ Oral Medicine \\ Division, Department of Diagnostic Oral Sciences, Faculty of Dentistry, King Abdulaziz University, Jeddah, Saudi \\ Arabia
}

\begin{abstract}
Introduction: Infective endocarditis is a serious infection of the heart endothelium and valves. It carries longterm health risks and compromises the heart condition. However, this condition has been rarely observed since the introduction of appropriate antibiotic prophylaxis. Dentists play a major role in preventing infective endocarditis in susceptible patients. In this study, we assessed the levels of awareness about antibiotic prophylaxis for infective endocarditis among students and young dentists at King Abdulaziz University, Jeddah, Saudi Arabia. Methods: This cross-sectional study included 367 dental students and dental interns who answered a questionnaire designed to assess their awareness levels; it included questions pertaining to demographic information and questions examining the general knowledge of the participants with regard to antibiotic prophylaxis for infective endocarditis. Results: An average of 50\% of the participants clearly lacked knowledge regarding the conditions that require antibiotic prophylaxis, while an average of $65 \%$ provided correct answers pertaining to the conditions that do not require prophylaxis. Regarding dental procedures that require prophylaxis, the majority of responses were correct with an average of $71.2 \%$. With regard to the type of antibiotic to be prescribed, $63.5 \%$ of the participants chose to prescribe $2 \mathrm{~g}$ of amoxicillin as a first-line antibiotic. Conclusion: The results of this study showed that the levels of awareness about antibiotic prophylaxis for infective endocarditis are below $100 \%$. Awareness is essential because of the cardiac risks associated with the lack of appropriate treatment. Up-to-date and accurate knowledge is mandatory for all dental students and interns who see and treat patients on a daily basis.
\end{abstract}

Keywords: Antibiotics, dental students, endocarditis, prophylaxis, Saudi Arabia, teaching hospital.

\section{INTRODUCTION}

Infective endocarditis (IE) is an infection of the endocardium caused by bacteria or fungi. It is a serious disease with a significant risk of morbidity and mortality [1]. Patients at risk of developing this disease include those with prosthetic heart valves, congenital heart defects, or a recent history of IE [1]. Bacterial endocarditis can occur following bacteraemia in patients with predisposing cardiac lesions [2].

The administration of prophylactic antibiotics before any interventional procedure that may lead to transient bacteraemia in patients at risk has been an accepted practice over the past 50 years [2]; however; the benefits of these antibiotics remain controversial. The guidelines of the British Society for Antimicrobial Chemotherapy and the American Heart Association (AHA) debate the need for prophylactic antibiotics by highlighting the prevalence of bacteraemia that arises from daily activities such as tooth brushing, the lack of an association between episodes of IE and prior interventional procedures, and the lack of efficacy of antibiotic prophylaxis regimens $[3,4]$.

*Address correspondence to this author at the P.O. Box 80209 Jeddah 21589 Kingdom of Saudi Arabia; Tel: +966505303227;

Fax: +966126403316; E-mail: mbahammam@kau.edu.sa
According to the AHA guidelines, individuals who are at risk of developing IE following an invasive dental procedure still benefit from antibiotic prophylaxis, while the guidelines of the National Institute for Health and Clinical Excellence in England and Wales recommend that prophylactic antibiotic treatment is no longer required for any at-risk patient. Bacteraemia following daily routines such as eating and tooth brushing may be a greater risk factor for the development of IE than the transient bacteraemia that follows an invasive dental procedure [5].

A recent study in 2012 showed no significant increase in the prevalence of IE caused by Streptococcus viridans in the United States after publication of the 2007 AHA guidelines that markedly restricted the use of antibiotic prophylaxis in certain at-risk patients undergoing dental and other invasive procedures [6]. Unfortunately, data and information regarding the prevalence of IE in Saudi Arabia is sparse [7]. A previous study conducted with the dental staff at Taibah University, Madinah, Saudi Arabia concluded that although most of the staff members followed the AHA guidelines, several did not. It also emphasized the need for continuous education on this critical topic [8].

To emphasize the importance of acquiring high-level and up-to-date knowledge of this subject, we conducted a literature search to identify medical topics that are of high importance for dental students and found a recent survey involving 
dentists and interns that was conducted to identify internal medicine topics that are the most relevant for dental education. Coagulopathy and endocarditis were rated the highest by the dentists, while anaphylaxis was rated the highest by the interns. The largest number of different internal diseases mentioned by the dentists and interns could be categorized into cardiovascular diseases [9]. This shows the importance of thorough knowledge of recent guidelines regarding antibiotic prophylaxis for IE and cardiac problems in general, although the relevance of these topics should be confirmed by larger quantitative studies performed to develop guidelines related to the specific learning objectives for internal medicine in the dental curriculum.

From the above-mentioned perspectives, we conducted this study to evaluate the levels of awareness regarding antibiotic prophylaxis for IE among fourth, fifth, and final year students and interns (during the first clinical year after graduation from the dental school, dental interns work under supervision from faculty members) at King Abdulaziz University, Jeddah, Saudi Arabia. Unlike previous studies that mainly targeted dental staff and general dentists [8, 10-12], our study is the first, to the best of our knowledge, to include dental students and interns.

\section{MATERIALS AND METHODS}

Our study adopted a cross-sectional design including a sample of 367 dental students and interns from the Faculty of Dentistry at King Abdulaziz University, Jeddah, Saudi Arabia. The students belonged to the fourth, fifth and final years, which are the clinical years of the course. All participants answered a questionnaire to determine their knowledge regarding antibiotic prophylaxis for IE prior to dental treatment. Ethical approval and a waiver of consent were obtained from the Ethical Committee of the Faculty of Dentistry, King Abdulaziz University.

A structured questionnaire was designed and developed on the basis of a thorough literature review [2-4, 8, 10]. It was divided into five sections assessing different categories as follows: demographic data, including age, gender, specialty, and level of education; participants' awareness regarding the necessity of prescribing antibiotics for different cardiac conditions; participants' awareness of dental procedures that need antibiotic prophylaxis; knowledge of the type and dose of antibiotic to be prescribed, assessed using direct questions, and the participants' source of information, assessed using a direct question. The AHA guidelines were used as a reference to compare our results against [4], because the faculty of Dentistry at King Abdulaziz University follows these international guidelines.

Each participant received a questionnaire with a brief description about the research as well as its importance. Verbal informed consent was obtained before study initiation. Then, the students and interns received the questionnaires in classrooms during break times. The questionnaire was distributed by the authors. In total, 500 questionnaires were prepared, of which 394 were answered; the remaining participants were engaged in other activities during form distribution. Of the 394, 27 questionnaires had one or more incomplete responses and were excluded. Eventually, 367 completed questionnaires were included in the study.
Data were entered into the Statistical package SPSS 15.00 for Windows (SPSS Inc., Chicago, IL). To simplify the results, some categories were grouped as correct and incorrect answers. Descriptive statistics in the form of frequencies and means were calculated for all study variables.

\section{RESULTS}

Of the 367 participants, $47.7 \%$ were male $(n=175)$ and $52.3 \%$ were female $(n=192)$. There were $113(30.8 \%)$ interns, with 11 being graduates from other Egyptian universities and one being a graduate from a Jordan university. Among the students, $27.5 \%(n=101)$ were in their fourth year, $24.3 \%(\mathrm{n}=89)$ were in their fifth year, and $17.4 \%(\mathrm{n}=$ 64) were in their final year. With regard to participant age, $7.6 \%(\mathrm{n}=28)$ were 20 years old, $68 \%(\mathrm{n}=251)$ were 22 years old, and $24 \%(\mathrm{n}=88)$ were $24-26$ years old. The participant characteristics are summarized in Table $\mathbf{1}$.

The response rate (75\%) was very high; this might reflect the simplicity of the questionnaire design. As stated in the methods section, the questionnaire included three main sections; the first one assessed the participants' knowledge of cardiac conditions that require antibiotic prophylaxis, the second assessed the knowledge of dental procedures that require prior antibiotic prophylaxis in cardiac patients, and the third section assessed the knowledge of antibiotic doses and alternatives.

Ten different cardiac conditions were included in the survey as shown in Table 2 . The results were divided according to conditions requiring or not requiring antibiotic prophylaxis according to the AHA guidelines. The total percentages of correct, incorrect and "I don't know" responses were calculated for each condition. An average of $50 \%$ $(41.4 \%-62 \%)$ of the participants clearly lacked knowledge regarding the conditions that require antibiotic prophylaxis, while $65 \%(59 \%-70.6 \%)$ provided correct answers pertaining to the conditions that do not require prophylaxis.

A total of 15 different dental procedures were mentioned in the second section, and the participants were questioned if these procedures required prior antibiotic prophylaxis according to the AHA guidelines. Table 3 shows the percentage of responses for each procedure; the majority of responses were correct.

With regard to the third section of the questionnaire, $63.5 \%(n=234)$ of the participants chose to prescribe $2 \mathrm{~g}$ of amoxicillin as a first-line antibiotic.

The last question was regarding the knowledge source of the participants: $27.8 \%(\mathrm{n}=102)$ used internet resources, $71.9 \%(\mathrm{n}=264)$ used the knowledge gained through lectures attended during under-graduation, and 34.3\% $(n=126)$ used knowledge obtained through information sharing with colleagues. All participants in this study reported the use of the AHA guidelines.

\section{DISCUSSION}

IE is a rare illness with a poor prognosis [13]. Considering the morbidity and the cost of treatment associated with the disease, dentists should have thorough and accurate knowledge of preventive measures. With regard to antibiotic 
Table 1. Participant characteristics.

\begin{tabular}{|c|c|c|c|c|c|c|c|c|c|}
\hline & \multicolumn{2}{|c|}{ Gender } & \multicolumn{3}{c|}{ Age Groups } & \multicolumn{4}{c|}{ Education Year } \\
\hline & Male & Female & $\mathbf{2 0}$ years & $\mathbf{2 2}$ years & $\mathbf{2 4 - 2 6}$ years & $\mathbf{4}^{\text {th }}$ year & $\mathbf{5}^{\text {th }}$ year & $\mathbf{6}^{\text {th }}$ year & Intern $^{\text {year }}$ \\
\hline \hline No. & 175 & 192 & 28 & 251 & 88 & 101 & 89 & 64 & 113 \\
\hline$\%$ & 47.7 & 52.3 & $7.6 \%$ & $68 \%$ & $24 \%$ & $27.5 \%$ & $24.3 \%$ & $17.4 \%$ & $30.8 \%$ \\
\hline
\end{tabular}

Table 2. A breakdown of the participants' responses to the first section regarding their knowledge of cardiac conditions that require prophylactic antibiotics.

\begin{tabular}{|c|c|c|c|}
\hline Ten cardiac conditions included in the survey & \multicolumn{3}{|c|}{ Responses } \\
\hline Prosthetic cardiac valves & $61 \%(225)$ & $33.5 \%(123)$ & $5.2 \%(19)$ \\
\hline Previous infective endocarditis & $62.1 \%(228)$ & $33.8 \%(124)$ & $4.1 \%(15)$ \\
\hline Residual defects after repair of CHDs & $41.4 \%(152)$ & $43.1 \%(158)$ & $15.5 \%(57)$ \\
\hline Untreated cyanotic congenital heart disease & $42 \%(157)$ & $34.6 \%(127)$ & $22.6 \%(83)$ \\
\hline Use of prosthetic materials for cardiac valve repair & $44.7 \%(164)$ & $37.3(137)$ & $18 \%(66)$ \\
\hline Repaired CHDs with no residual defects at the site & $70.3(258)$ & $8.2 \%(30)$ & $21.5 \%(79)$ \\
\hline Heart murmurs & $70.6(259)$ & $6.8 \%(25)$ & $22.6 \%(83)$ \\
\hline Heart failure & $59.9 \%(220)$ & $15.8 \%(58)$ & $24.3 \%(89)$ \\
\hline
\end{tabular}

AHA, American heart association; CHDs, congenital heart defect

prophylaxis before dental intervention, different guidelines have been followed in different countries, with the most followed international guidelines being the National Institute of Clinical Excellence (NICE) guidelines [2] and the AHA guidelines.

The dental school at King Abdulaziz University teaches the AHA guidelines to under-graduates and ensures that all employees follow the same guideline. In the present study, we assessed the level of awareness about antibiotic prophylaxis for IE among fourth, fifth, and final year dental students, who see and treat patients on a daily basis, as well as dental interns. All participants were students or interns at King Abdulaziz University; only 12 interns had graduated from other universities in Egypt and Jordan. Our questionnaire was short and quick, and this was reflected by the high response rate of the participants $(75 \%)$. Furthermore, all the included interns were motivated because of the importance of the subject and its impact on patient care. The number of female participants in this study was higher $(52.3 \%$ vs. $47.7 \%$ ), and this is because the number of female students in all academic years in our school is higher. The age range of the participants was 20-26 years, consistent with the levels of education, with $68 \%$ participants aged 22 years.
Our results showed that all participants reported the use of the AHA guidelines. This was expected because all the health-care facilities and dental schools in Saudi Arabia use these guidelines. A total of $71.9 \%(n=264)$ of the participants reported that they gained their knowledge of this subject through lectures or clinical training conducted during the under-graduate course. This can be explained by the fact that all our participants were students or interns. The remaining $27.8 \%(\mathrm{n}=102)$ used internet resources and 34.3\% (126) gained their knowledge through information-sharing with colleagues.

With regard to the first section of our questionnaire, twothirds of the participants were aware that patients with prosthetic heart valves and previous IE do need antibiotic prophylaxis prior to dental treatment, while only $41 \%-55 \%$ showed awareness regarding the requirements for cases involving surgically repaired or unrepaired congenital heart defects. This was probably because the guidelines were recently revised and recommended that congenital conditions no longer require antibiotic prophylaxis, while guidelines for the first two conditions remained unchanged. A small percentage of participants $(11 \%-22 \%)$ were unsure of their answers. With regard to heart murmurs, $70.6 \%$ participants 
Table 3. A breakdown of the participants' responses to the second section regarding their knowledge of dental procedures requiring prophylactic antibiotics.

\begin{tabular}{|c|c|c|c|}
\hline Dental procedures & \multicolumn{3}{|c|}{ Responses } \\
\hline \multicolumn{4}{|c|}{ Procedures that do not require antibiotic prophylaxis according to the AHA guidelines } \\
\hline Supra-gingival scaling & $86 \%(319)$ & $9.5 \%(35)$ & $3.5 \%(13)$ \\
\hline Class II fillings & $60.5 \%(222)$ & $30.5 \%(112)$ & $9 \%(33)$ \\
\hline Root canal treatment & $35.1 \%(129)$ & $61.3 \%(225)$ & $3.5 \%(13)$ \\
\hline Routine anaesthetic injections through non-infected tissue & $75.5 \%(277)$ & $18.8 \%(69)$ & $5.7 \%(21)$ \\
\hline Dental radiography & $95.5 \%(352)$ & $2.2 \%(8)$ & $1.9 \%(7)$ \\
\hline Placement of removable prosthodontic or orthodontic appliances & $80.9 \%(297)$ & $9.8 \%(36)$ & $9.3 \%(34)$ \\
\hline Shedding of deciduous teeth & $76 \%(279)$ & $3.3 . \%(12)$ & $20.7 \%(76)$ \\
\hline Bleeding from trauma to the lips or oral mucosa & $41 \%(152)$ & $45.5 \%(167)$ & $13.1 \%(48)$ \\
\hline \multicolumn{4}{|c|}{ Procedures that require antibiotic prophylaxis according to the AHA guidelines } \\
\hline Forceps extractions & $75.5 \%(277)$ & $18.3 \%(67)$ & $5.8 \%(23)$ \\
\hline Implant placement & $75.2 \%(276)$ & $13.9 \%(51)$ & $10.9 \%(40)$ \\
\hline Sub-gingival scaling & $70.3 \%(258)$ & $22.6 \%(83)$ & $7.1 \%(26)$ \\
\hline Surgical extractions & $86.4 \%(317)$ & $9.8 \%(36)$ & $3.8 \%(14)$ \\
\hline
\end{tabular}

provided correct answers, while only $59.9 \%$ provided correct answers with regard to heart failure, probably because of revision of the old guidelines.

With regard to the second section, the majority of participants $(75 \%-86 \%)$ correctly reported that forceps extractions, implant placement, sub-gingival scaling, and surgical extractions require prior antibiotic prophylaxis. Although the majority of participants $(84 \%)$ provided correct answers in this section, several provided incorrect answers for root canal treatment $(\mathrm{n}=61 ; 3 \%)$, possibly because of confusion regarding periapical involvement and pulp exposure.

As stated previously, bacteraemia following daily routines such as eating and tooth brushing may be a greater risk factor for the development of IE than the transient bacteraemia that follows an invasive dental procedure [5]; this led to the recent changes in the AHA guidelines. Dentists and health-care professionals are obliged to update their knowledge regarding this subject to maintain high standards of care. Dentists should always follow the guidelines that are enforced in their hospitals. The results of our study were similar to those of another study conducted at Taibah University, Madinah, Saudi Arabia [8], although the previous study included dental staff members rather than students or interns. The authors recommended that nationally standardized guidelines should be discussed and implemented across the kingdom to ensure that all students and patients receive the most appropriate and acceptable treatment modalities [7].

\section{CONCLUSION AND RECOMMENDATION}

The results of this study showed that the levels of awareness about antibiotic prophylaxis for IE have not reached $100 \%$ among dental students and interns. Considering the importance of good knowledge about antibiotic prophylaxis for IE prior to dental treatment and the cardiac risks associated with the lack of appropriate treatment, $100 \%$ accurate knowledge should be mandatory for all dental students and interns who deal with patients on a daily basis. Guidelines should be reinforced several times in the under-graduate years as well as during internship. This can be achieved through continuous lectures or seminars. In addition, students and interns must be assessed for their knowledge at different levels and all staff members (lecturers and professors) must remain updated.

\section{CONFLICT OF INTEREST}

The authors confirm that there are no conflicts of interest associated with this publication. There has been no significant financial support that could have influenced the outcome of this study. This project was funded by the Deanship of Scientific Research (DSR), King Abdulaziz University, Jeddah under grant No. 287-254-1434.

\section{ACKNOWLEDGEMENTS}

This project was funded by the Deanship of Scientific Research (DSR), King Abdulaziz University, Jeddah under 
grant No. 287-254-1434. The authors acknowledge this technical and financial support.

\section{REFERENCES}

[1] Pierce D, Calkins BC, Thornton K. Infectious endocarditis: diagnosis and treatment. Am Fam Physician 2012; 85: 981-6.

[2] Center for Clinical Practice at Nice. Prophylaxis against infective endocarditis: antimicrobial prophylaxis against infective endocarditis in adults and children undergoing interventional procedures. London: National Institute for Health and Clinical Excellence (UK) 2008.

[3] Gould FK, Elliott TS, Foweraker J, et al. Guidelines for the prevention of endocarditis: report of the Working Party of the British Society for Antimicrobial Chemotherapy. J Antimicrob Chemother 2006; 57: 1035-42.

[4] Wilson W, Taubert KA, Gewitz M, et al. Prevention of infective endocarditis: guidelines from the American Heart Association: a guideline from the American Heart Association Rheumatic Fever, Endocarditis and Kawasaki Disease Committee, Council on Cardiovascular Disease in the Young, and the Council on Clinical Cardiology, Council on Cardiovascular Surgery and Anesthesia, and the Quality of Care and Outcomes Research Interdisciplinary Working Group. J Am Dent Assoc 2008; 139: 3S-24S.

[5] Costantinides F, Clozza E, Ottaviani G, et al. Antibiotic prophylaxis of infective endocarditis in dentistry: clinical approach and controversies. Oral Health Prev Dent 2014; 12(4): 305-11.
[6] Desimone DC, Tleyjeh IM, Correa De Sa DD, et al. Incidence of infective endocarditis caused by viridans group streptococci before and after publication of the 2007 American Heart Association's endocarditis prevention guidelines. Circulation 2012; 126: 60-4.

[7] Al-Tawfiq JA, Sufi I. Infective endocarditis at a hospital in Saudi Arabia: epidemiology, bacterial pathogens and outcome. Ann Saudi Med 2009; 29: 433-6.

[8] Bhayat A, Jarab F, Mansuri S, Ahmad MS, Mahrous MS. Assessment of knowledge of dental staff at a saudi arabian university regarding the prophylaxis for infective endocarditis. Open Dent $\mathrm{J}$ 2013; 7: 82-7.

[9] Kunde A, Harendza S. Topics of internal medicine for undergraduate dental education: a qualitative study. Eur J Dent Educ 2014 [Epub Ahead of Print]

[10] Adeyemo WL, Oderinu OH, Olojede AC, Ayodele AO, Fashina AA. Nigerian dentists' knowledge of the current guidelines for preventing infective endocarditis. Commun Dent Health 2011; 28: 178-81.

[11] Ahmadi-Motamayel F, Vaziri S, Roshanaei G. Knowledge of general dentists and senior dental students in Iran about prevention of infective endocarditis. Chonnam Med J 2012; 48: 15-20.

[12] Nakano K, Ooshima T. Common knowledge regarding prevention of infective endocarditis among general dentists in Japan. J Cardiol 2011; 57: 123-30.

[13] Klein M, Wang A. Infective endocarditis. J Intensive Care Med 2014. [Epub Ahead of Print]

This is an open access article licensed under the terms of the Creative Commons Attribution Non-Commercial License (http://creativecommons.org/licenses/by-nc/3.0/) which permits unrestricted, non-commercial use, distribution and reproduction in any medium, provided the work is properly cited. 